\title{
Long-Term Efficacy Analysis of Exclusive Enteral Nutrition for the Treatment of Abdominal Abscess in Crohn's Disease
}

\author{
Ni Ding, Qingfan Yang, Huiping Chen, Mengting Hu, Hong Yan, Xiang Gao* \\ Department of Gastroenterology, The Sixth Affiliated Hospital of Sun Yat-sen University, Guangzhou, China \\ Email: dingn8@mail.sysu.edu.cn
}

How to cite this paper: Ding, N., Yang, Q.F., Chen, H.P., Hu, M.T., Yan, H. and Gao, X. (2021) Long-Term Efficacy Analysis of Exclusive Enteral Nutrition for the Treatment of Abdominal Abscess in Crohn's Disease. International Journal of Clinical Medicine, 12, 342-350.

https://doi.org/10.4236/ijcm.2021.128030

Received: July 13, 2021

Accepted: August 13, 2021

Published: August 16, 2021

Copyright @ 2021 by author(s) and Scientific Research Publishing Inc. This work is licensed under the Creative Commons Attribution International License (CC BY 4.0).

http://creativecommons.org/licenses/by/4.0/ (c) (i) Open Access

\begin{abstract}
Objective: The objective is to explore the long-term efficacy of total enteral nutrition in the treatment of abdominal abscess in Crohn's disease. Methods: Patients treated with EEN in our hospital and whose abdominal abscess disappeared after 12 weeks of treatment were included, and the data of abscess recurrence and surgical treatment during follow-up were included. Results: A total of 44 consecutive cases meeting the criteria were included. The 1-year and 2 -year recurrence rates were $22.5 \%$ and $39.9 \%$ respectively. Among the patients with recurrence, 10 patients chose EEN treatment again, and 5 patients received direct surgical treatment. Of the patients who chose to undergo EEN treatment again, 8 still eventually required surgery. The 1-year operative rate was $16.9 \%$, and the 2 -year operative rate was $35.6 \%$. The median operative time was 33.3 (95\% CI: 21.3, 45.4) months. Conclusions: The 2-year cumulative incidence of recurrence of abdominal abscess in $\mathrm{CD}$ patients whose abdominal abscess disappeared after EEN was $39.9 \%$, and the 2-year cumulative surgical rate was $35.6 \%$. The operative stomy rate decreased after EEN, and the primary anastomosis rate increased significantly.
\end{abstract}

\section{Keywords}

Crohn's Disease, Enteral Nutrition, Abdominal Abscess, Long-Term Efficacy

\section{Introduction}

Crohn's disease (CD) can cause serious complications such as intestinal fistula, abdominal abscess, and intestinal stenosis during the course of disease. Studies showed that the cumulative incidence of $\mathrm{CD}$ complicated with intestinal fistula/abdominal abscess was up to $33 \%$ in 10 years after diagnosis, and up to $50 \%$ after 20 years of diagnosis [1]. Our data suggest that $\mathrm{CD}$ with abdominal abscess 
accounts for about $47 \%$ of penetrating CD patients, indicating that abdominal abscess represents one of the common penetrating behaviors. Currently, anti-infective therapy is the primary choice for the treatment of CD patients with abdominal abscess, and the requirement for percutaneous drainage is determined according to abscess size and location [2]. Previous studies found that, after anti-infection and percutaneous drainage, $50 \%-83 \%$ of the patients still required surgical drainage of the abscess and removal of the affected intestine, and the postoperative recurrence rate of intestinal fistula/abdominal abscess was up to $13 \%$ [3] [4] [5] [6] [7]. In addition, because these patients are often in the active stage of moderate to severe disease accompanied by malnutrition, conventional remission induction regimens such as hormones or biologics may aggravate the progression of this disease. Moreover, these patients often do not need emergency surgery or cannot tolerate surgery due to malnutrition. Therefore, there are certain difficulties and challenges in the treatment of CD patients with abdominal abscess.

Guideline on Enteral Nutrition of the European Society for Parenteral Enteral Nutrition (ESPEN) points out: for other patients, the main role of enteral nutrition is to improve the nutritional state and promote growth and development; for CD patients, enteral nutrition has special significance: it can be used for acute phase therapy, perioperative nutrition, and maintenance of remission. In current studies on exclusive enteral nutrition (EEN) for the treatment of CD, however, few literatures reported the value of EEN in the treatment of abdominal abscess. The results of a recent prospective observational study at our site [8] suggest that a 12-week EEN therapy can safely and effectively facilitate clinical remission, mucosal healing, reduction of inflammatory indexes, imaging remission, and reduction of surgical rate in active CD patients with abdominal abscess.

In recent years, in order to avoid surgery and occurrence of postoperative complications, the gastroenterologists proposed "SNAP" (sepsis and skin care, nutritional support, definition of anatomy and surgical procedure) treatment strategy. This strategy recommends that, for patients with abdominal abscess or intestinal fistula, it should extend the conservative treatment time and postpone the operation as much as possible. It is recommended that emergency treatment, including anti-infection, maintenance of water and electricity balance, pain relief and skin care, be given first, followed by nutritional therapy and efficacy evaluation. After which, the anatomy of gastrointestinal tract should be clarified, and surgical treatment can be performed if necessary. In the study on EEN for the treatment of CD with abdominal abscess conducted at our site as mentioned above [8], all therapeutic procedures followed the SNAP strategy, the patients were provided with necessary anti-infection, skin care, and EEN for 12 weeks, and meanwhile the intestinal conditions were timely assessed to determine the necessity of surgery. Abdominal abscess disappeared in $76 \%$ of the patients after treatment. This indicates that SNAP strategy and EEN are clearly effective on CD complicated with abdominal abscess. 
However, current studies on EEN for the treatment of CD patients with abdominal abscess, including the results of previous studies of this site, have paid more attention to short-term efficacy. Further observation and research are needed to determine the long-term outcomes of patients who have achieved clinical remission of $\mathrm{CD}$ and shrinkage or disappearance of the abscess after short-term EEN therapy.

Therefore, this study was designed to retrospectively analyze the clinical data of $\mathrm{CD}$ patients with spontaneous abdominal abscess after EEN therapy in our hospital from January 1, 2014 to December 1, 2017 in order to explore the long-term efficacy of EEN on CD patients with abdominal abscess.

\section{Subjects and Methods}

\subsection{Study Subjects}

Patients who received EEN from January 1, 2014 to December 1, 2017 in this hospital and the abdominal abscess disappeared after 12 weeks of treatment (regardless of whether there was active CD or continuing medication).

Inclusion Criteria:

1) Patients aged 18 - 60 years, male or female;

2) Confirmed CD diagnosis, CTE/MRE-measured long diameter of abdominal abscess $\geq 1 \mathrm{~cm}$, CTE/MRE-confirmed disappearance of abdominal abscess after 12 weeks of EEN therapy;

3) EEN has been discontinued.

Exclusion Criteria:

1) Children, pregnant and lactating patients;

2) Newly added hormones/biologics/immunosuppressants during EEN (excluding hormones/biologics/immunosuppressants administered before EEN);

3) Surgical operations within the imminent period of abdominal abscess (3 months), suspected surgery-related abscess.

\subsection{Study Methods}

This single-center retrospective study included a total of 44 consecutive cases meeting the criteria. In the cases of abscess recurrence or surgery, the patients were observed until abscess recurrence/surgery has occurred; for patients without recurrence, the observation continued until October 1, 2019. The primary observation endpoints of this study were the recurrence rate and time of abdominal abscess (abscess recurrence rate and the time from initial recurrence to EEN discontinuance were calculated). In addition, it was necessary not only to clarify surgical rate and method (one-stage anastomosis or enterostomy) of patients after EEN but also to specify the time from surgical operation to EEN discontinuance. Collection time points included: the beginning of EEN, 12 weeks of treatment, abscess recurrence/operation after EEN discontinuance, and follow-up to the most recent comprehensive reexamination for patients without recurrence.

The data were collected by retrieving inpatient electronic medical record sys- 
tem of our hospital, and the collection time points included: the beginning of EEN, 12 weeks of treatment, abscess recurrence/operation after EEN discontinuance, and follow-up to the most recent comprehensive reexamination for patients without recurrence. The collected data included demographic data, CD course, CD clinical classification, and imaging data (combined enterocutaneous fistula, intestinal fistula, intestinal adhesion and entanglement, and intestinal stenosis).

The relevant definitions of this study were as follows:

Spontaneous abdominal abscess was defined as the abdominal abscess that had a diameter $\geq 1 \mathrm{~cm}$ determined by imaging and was caused by inflammatory penetration, unrelated to intestinal surgery, but enterocutaneous fistula/abdominal abscess emergent within 3 months after the operation was considered to be surgery-related. Abscess recurrence was defined as the recurrent abscess at the same site as before treatment.

\subsection{Statistical Methods}

All involved quantitative data were subject to $t$-test, and all qualitative data were subject to chi-square test. $\mathrm{P}<0.05$ was considered to have statistical difference. Kaplan-Meier method was used to calculate the long-term recurrence rate and surgical rate of the abscess. All data were analyzed and processed using SPSS 25.0 (SPSS, Chicago, IL) statistical software.

\section{Results}

From January 1, 2014 to December 30, 2017, this site included a total of $44 \mathrm{CD}$ patients with spontaneous abdominal abscess in this study for regular follow ups. Table 1 summarizes the detailed demographic characteristics of the patients. The median follow-up time for all patients was $26.3(13.3,42.8)$ months.

\subsection{Long-Term Abscess Recurrence Rate in CD Patients Whose Abdominal Abscess Disappeared after 12 Weeks of EEN Therapy}

By the end of this study, a total of 16 patients (36.4\%) experienced recurrence of abdominal abscess, among them, the recurrence occurred at other site in 1 case (2.3\%) and at the same site in 15 cases (34.1\%). The 1-year abscess recurrence rate was $22.5 \%$, the 2 -year abscess recurrence rate was $39.9 \%$, and the median recurrence time was 63.4 (95\% CI: 13.8, 113.0) months. The cumulative recurrence rate of abscess was shown in Figure 1.

\subsection{Long-Term Abscess Surgical Rate in CD Patients Whose Abdominal Abscess Has Disappeared after 12 Weeks of EEN Therapy}

By the end of this study, a total of 20 patients (45.5\%) had undergone surgical treatment. The 1-year operation rate was $16.9 \%$, the 2 -year operation rate was 
$35.6 \%$, and the median operation time was 33.3 (95\% CI: 21.3, 45.4) months. Patient's long-term cumulative surgical rate was shown in Figure 2.

Table 1. All basic demographic data $(n=44)$.

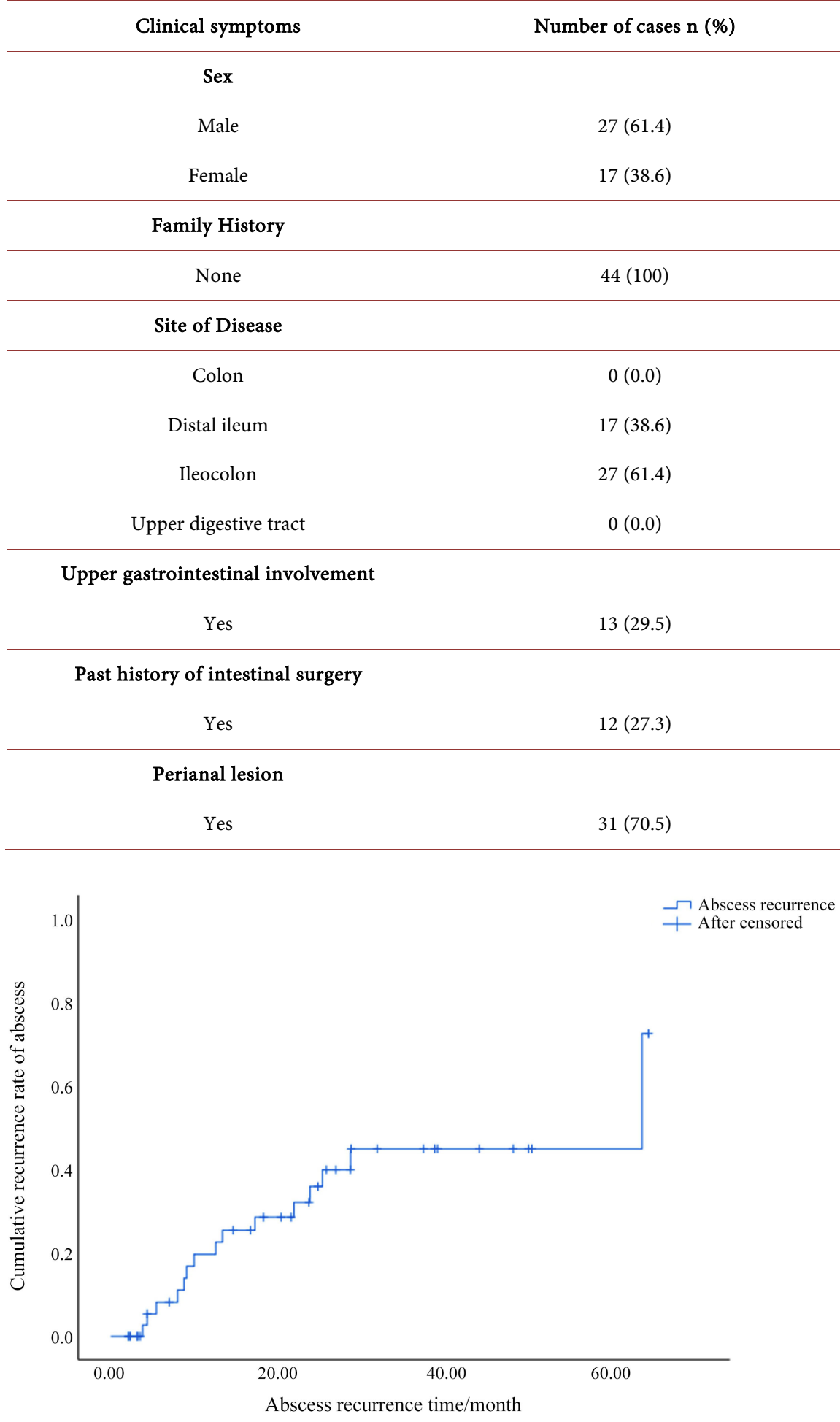

Figure 1. Cumulative recurrence rate of abscess in CD patients whose abdominal abscess has disappeared after 12 weeks of EEN therapy. 


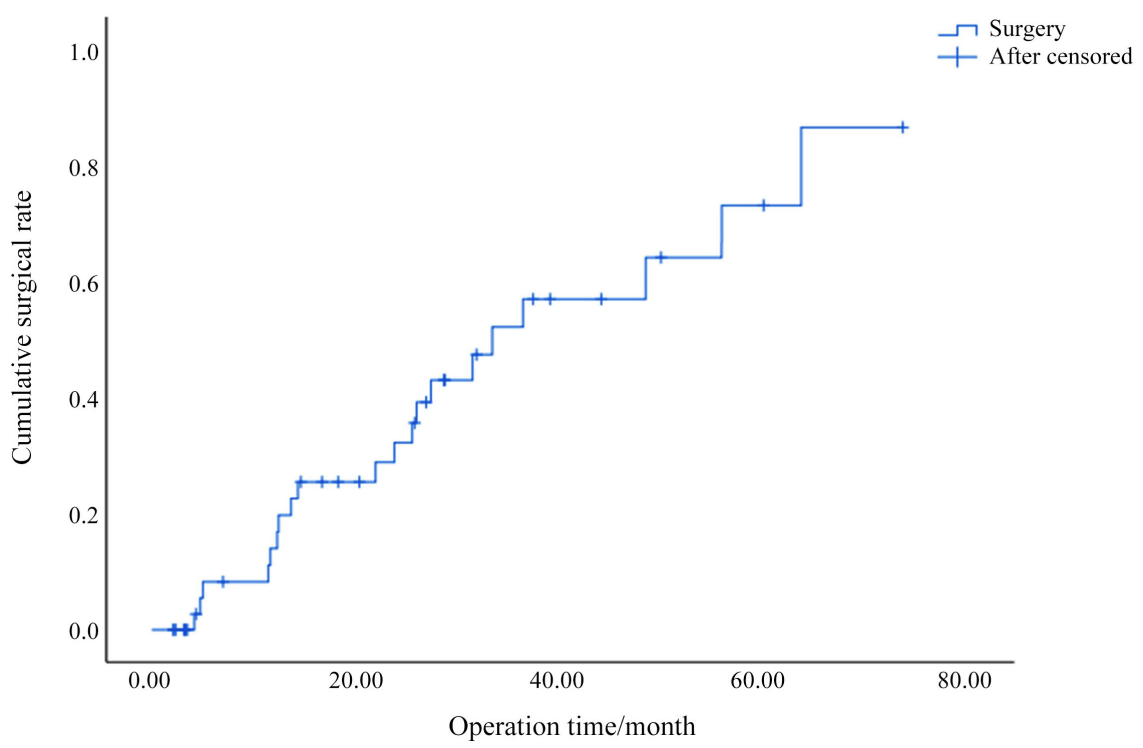

Figure 2. Cumulative surgical rate of CD patients whose abdominal abscess has disappeared after 12 weeks of EEN therapy.

\subsection{Enterostomy Rate Following Recurrence in CD Patients Whose Abdominal Abscess Has Disappeared after 12 Weeks of EEN Therapy}

By the end of the study, a total of 15 patients (34.1\%) experienced abscess recurrence, among them, 13 patients $(29.5 \%)$ switched to surgical treatment as the final outcome, and only 2 patients (4.5\%) avoided surgery after active medication.

Among all 15 patients experiencing abscess recurrence, 10 cases $(66.7 \%)$ tried the second round of EEN, followed by further evaluation of the intestinal lesions, including 2 cases (13.3\%) avoiding surgery and 8 cases (53.3\%) eventually receiving surgical treatment; the remaining 5 patients (33.3\%) directly underwent surgery without receiving the second round of EEN.

We included the 13 patients who finally underwent surgery after abscess recurrence as the study subjects and grouped them by EEN following abscess recurrence in order to compare enterostomy between the 2 groups of patients (see Table 2). The results showed that the proportion of enterostomy was lower in patients receiving EEN after abscess recurrence than in patients directly undergoing surgery without receiving EEN therapy, and the difference had statistical difference $(\mathrm{P}<0.05)$.

Table 2. Comparison of enterostomy rate following recurrence after CD patients with spontaneous abdominal abscess have been cured by EEN.

\begin{tabular}{cccc}
\hline & With enterostomy & Without enterostomy & P \\
\hline $\begin{array}{c}\text { Direct operation } \\
\text { after recurrence }\end{array}$ & $4(80.0)$ & $1(12.5)$ & $\mathbf{0 . 0 3 2}$ \\
$\begin{array}{c}\text { Operation after repeated } \\
\text { EEN following recurrence }\end{array}$ & $1(20.0)$ & $7(87.5)$ & \\
\hline
\end{tabular}




\section{Discussion}

Abdominal abscess is a common serious complication during the course of CD. The current opinions on its treatment principle are anti-infection plus abscess drainage, and enterectomy if the patients are unresponsive to the above treatment [1] [9]; because patients with active CD often have concomitant malnutrition, it is necessary to actively improve their nutritional state and control CD inflammatory activities before surgery in order to reduce the risk of postoperative complications [10]. As mentioned above, a recent prospective study at our site suggests that EEN is able to achieve clinical remission, mucosal healing, reduction of inflammatory indexes, imaging remission, and reduction of surgical rate [8]. In the Chinese Consensus on Nutritional Treatment of Inflammatory Bowel Disease (2018), it is clearly stated that EN is the primary choice for CD patients with abdominal abscess/intestinal fistula under the prerequisite of adequate drainage, and surgery can be avoided in some simple small intestinal fistulas that may heal spontaneously after EN or PN therapy [11]. Therefore, EEN is an effective treatment protocol for CD complicated with abdominal abscess; it can not only be used as a preoperative preparation for patients requiring surgical intervention but also cure abdominal abscess in patients who do not need surgery. However, the long-term recurrence rate and surgical rate of $\mathrm{CD}$ complicated with abdominal abscess cured after short-term EEN therapy are still unknown, and no clear report is readily available. This part of the study aimed to determine the long-term abscess recurrence rate and surgical rate of CD patients with spontaneous abdominal abscess cured after EEN therapy.

Our study results showed that, by the end of this study, 16 of the $44 \mathrm{CD}$ patients whose abdominal abscesses disappeared after EEN therapy experienced abscess recurrence, among which, the abscess recurred at the same site in $15 \mathrm{pa}-$ tients, and the abscess recurred at other sites in 1 patient. The 1-year abscess recurrence rate was $22.5 \%$, the 2-year abscess recurrence rate was $39.9 \%$, and the median recurrence time was 63.4 (95\% CI 12.8, 113.0). Among these recurrent cases, 10 patients selected repeated EEN, and 5 patients received direct surgery; 8 of the patients selecting repeated EEN eventually needed surgical intervention. Therefore, 13 patients eventually underwent surgery, the 1-year operation rate was $16.9 \%$, the 2 -year operation rate was $35.6 \%$, and the median operation time was 33.3 (95\% CI 21.3, 45.4) months. Among the patients undergoing surgery, 8 cases selected one-stage anastomosis, and 12 cases received enterostomy, in staged operations; patients undergoing surgery after EEN mostly selected one-stage operation, while patients undergoing direct surgery after the recurrence of abdominal abscess more often selected staging operations. The difference between these 2 groups had statistical significance $(\mathrm{P}<0.05)$, suggesting that EEN was a protective factor for one-stage surgery. Currently, there are few studies on the long-term efficacy of EEN on CD complicated with abdominal abscess at home and abroad. A recent randomized and controlled study compared the effect of EEN on postoperative complications in adult CD patients using nutritional and 
inflammatory indexes as the preoperative nutritional therapy endpoints in China. The results showed that both nutritional indexes and inflammatory indexes were improved in patients undergoing surgery after EEN. In the meantime, EEN therapy significantly reduced enterostomy rate and the incidence of postoperative complication in these patients [12]. Our study findings were similar to the results of this study, and EEN indeed reduced the surgical rate in these patients. The long-term surgical rate after EEN was $45.5 \%$, the recurrence rate was $34.1 \%$, and the enterostomy rate after EEN was $40 \%$. The one-stage anastomosis rate significantly increased, while postoperative complications decreased. In summary, this part of the study still has some shortcomings; it is necessary to increase the number of cases not only to further confirm the current conclusions but also to analyze the risk factors related to the long-term recurrence rate and surgical rate.

In conclusion: The 2-year recurrence rate of $\mathrm{CD}$ complicated with spontaneous abdominal abscess cured after EEN is $39.9 \%$, and the 2-year surgical rate is $35.6 \%$. After EEN, enterostomy rate decreases, while one-stage anastomosis rate significantly increases.

\section{Conflicts of Interest}

The authors declare no conflicts of interest regarding the publication of this paper.

\section{References}

[1] Papi, C., et al. (2005) Evolution of Clinical Behaviour in Crohn's Disease: Predictive Factors of Penetrating Complications. Digestive and Liver Disease, 37, 247-253. https://doi.org/10.1016/j.dld.2004.10.012

[2] IBD Group of the Society of Gastroenterology, Chinese Medical Association (2018) Consensus on the Diagnosis and Treatment of Inflammatory Bowel Disease (2018, Beijing). Chinese Journal of Digestion, 38, 292-311.

[3] Alkhouri, R.H. et al. (2017) Outcome of Medical Management of Intraabdominal Abscesses in Children with Crohn Disease. Journal of Pediatric Surgery, 52, 1433-1437. https://doi.org/10.1016/j.jpedsurg.2017.03.059

[4] Gervais, D.A., et al. (2002) Percutaneous Abscess Drainage in Crohn Disease: Technical Success and Short- and Long-Term Outcomes during 14 Years. Radiology, 222, 645-651. https://doi.org/10.1148/radiol.2223010554

[5] Garcia, J.C., et al. (2001) Abscesses in Crohn's Disease: Outcome of Medical Versus Surgical Treatment. Journal of Clinical Gastroenterology, 32, 409-412. https://doi.org/10.1097/00004836-200105000-00010

[6] Bermejo, F., et al. (2012) Efficacy of Different Therapeutic Options for Spontaneous Abdominal Abscesses in Crohn's Disease: Are Antibiotics Enough? Inflammatory Bowel Diseases, 18, 1509-1514. https://doi.org/10.1002/ibd.21865

[7] Takayuki, Y., et al. (2000) Risk Factors for Intra-Abdominal Sepsis after Surgery in Crohn's Disease. Diseases of the Colon \& Rectum, 43, 1141-1145. https://doi.org/10.1007/BF02236563

[8] Yang, Q.F., et al. (2017) Efficacy of Exclusive Enteral Nutrition in Complicated Crohn's Disease. Scandinavian Journal of Gastroenterology, 52, 995-1001.

[9] Gomollón, F., et al. (2017) 3rd European Evidence-Based Consensus on the Diag- 
nosis and Management of Crohn's Disease 2016: Part 1: Diagnosis and Medical Management. Journal of Crohn's and Colitis, 11, 3-25.

[10] Chinese IBD Clinical Trial Group (2015) Expert Opinions on the Risk Factors and Prevention of Postoperative Complications of Inflammatory Bowel Disease (2014, Guangzhou). Chinese Journal of Gastrointestinal Surgery, 18, 388-394.

[11] IBD Group of the Society of Gastroenterology, Chinese Medical Association (2018) Expert Consensus on Nutritional Support Therapy of Inflammatory Bowel Disease (2nd Edition). Chinese Journal of Inflammatory Bowel Disease (Chinese and English), 2, 154-172.

[12] Zhu, W.M., et al. (2015) CONSORT: Different End-Points of Preoperative Nutrition and Outcome of Bowel Resection of Crohn Disease: A Randomized Clinical Trial. Medicine, 94, e1175. https://doi.org/10.1097/MD.0000000000001175 